P01.03 VOC PATTERN RECOGNITION OF LUNG CANCER: A COMPARATIVE EVALUATION OF DIFFERENT DOG- AND ENOSE-BASED STRATEGIES USING DIFFERENT SAMPLING MATERIALS

${ }^{1} W$ Biehl ${ }^{*},{ }^{1} H$ Schmetzer, ${ }^{2} R$ Koczulla, ${ }^{3} A$ Hattesohl, ${ }^{4} R$ Jörres, ${ }^{5} \mathrm{~T}$ Duell, ${ }^{6} U$ Althöhn. ${ }^{1}$ Medical Department III, University Hospital Großhadern, Ludwig-Maximilians-University, Munich, Germany; ${ }^{2}$ Department of Respiratory Medicine and Pulmonary Rehabilitation, Schoen Klinik Berchtesgadener Land, Schoenau am Koenigssee, Schönau am Königssee, Germany; ${ }^{3}$ Department of Pulmonary Rehabilitation, Philipps-University of Marburg, German Center for Lung Research, Marburg, Germany; ${ }^{4}$ Institute and Outpatient Clinic for Occupational, Social and Environmental Medicine, University Hospital, Ludwig-MaximiliansUniversity, Munich, Germany; ${ }^{5}$ Department for Oncology, Asklepios Lungenfachkliniken Muenich-Gauting, Munich, Germany; ${ }^{6}$ Evidensia Tierärztliche Klinik für Kleintiere Norderstedt GmbH, Norderstedt, Germany

\subsection{6/jitc-2020-ITOC7.16}

Background It has been reported that canine scent tests offer the possibility to screen for cancer. Assuming that breath samples can be collected with carrier materials, we tested the practicability of different carrier materials to be presented to dogs, and validated and compared results with an eNose. Moreover, we hypothesised that cancer detection ability of dogs differs according to their working experience.

Materials and Methods In a methodological approach two dog teams participated, one using experienced working dogs and the other ordinary household dogs to find discover which dogs were better qualified and the best training method. To find best carrier material for breath sampling we compared charcoal containing glass tubes with fleece masks. In a second validating part, experienced working dogs were trained with improved training strategies. For breath sampling two different, previously successfully tested fleece-based carrier materials were used: one was used with the dog team and both materials were compared with eNose.

Results In the first part of the study it was shown overall that experienced working dogs performed better to family dogs and the dogs achieved a sensitivity of $45-59 \%$ and a specificity of $45-69 \%$. Charcoal based breath sample carrier materials did not qualify for detection of VOC by dogs. In the second part of the study, the dogs achieved a specificity of $83 \%$ and a sensitivity of $56 \%$, but with considerable differences between individual dogs. The eNose provided a specificity of $97 \%$ for both fleece based carrier materials and a sensitivity of $89 \%$ for fleece filled glass tubes and $100 \%$ for earloop masks. Measurements of breath samples collected directly in respiratory bags as reference measurements achieved a sensitivity and specificity of $100 \%$.

Conclusions Our data confirmed that diagnostic accuracy of dogs depended on the type of dog training and on the carrier materials. A comparison of breath samples analysis with an eNose achieved better results for both, sensitivity and specificity, than for dogs. The use of fleece masks or fleeces in glass tubes as a sampling material can be recommended as successful VOC carriers, encouraging their use for clinical screenings.

Disclosure Information W. Biehl: None. H. Schmetzer: None. R. Koczulla: None. A. Hattesohl: None. R. Jörres: None. T. Duell: None. U. Althöhn: None.

\section{P01.04 A SPATIALLY RESOLVED, HIGHLY MULTIPLEXED BIOMARKER ANALYSIS PIPELINE THAT BRIDGES THE DIVIDE BETWEEN DISCOVERY AND CLINICAL RESEARCH}

${ }^{1} \mathrm{O}$ Braubach*, ${ }^{1} \mathrm{M}$ Gallina, ${ }^{2} \mathrm{~B}$ Remeniuk, ${ }^{2} \mathrm{C}$ Wang, ${ }^{1} \mathrm{~N}$ Nikulina, ${ }^{1} \mathrm{R}$ Bashier, ${ }^{1} \mathrm{~J}$ KennedyDarling, ${ }^{2} \mathrm{C}$ Hoyt. 'Akoya Biosciences, Menlo Park, CA, USA; ${ }^{2}$ Akoya Biosciences, Marlborough, MA, USA

\subsection{6/jitc-2020-ITOC7.17}

Background Multiplexed immunofluorescence (mIF) allows the visualization of multiple biomarkers in a single tumor tissue section, while at the same time preserving the spatial biology of the tumor microenvironment (TMI). CO-Detection by indEXing $\left(\mathrm{CODEX}^{\oplus}\right)$ and Phenoptics ${ }^{\mathrm{TM}}$ platforms are complementary mIF technologies that span the full spectrum of cancer research, from discovery to translational and clinical research. CODEX ${ }^{\circledast}$ is ultra-high plex and allows imaging of up to 40 antigens on a single tissue section with single-cell resolution. Phenoptics ${ }^{\mathrm{TM}}$ is an established mIF platform that enables high-throughput whole slide multispectral image acquisition and tissue interrogation with up to 8 markers plus DAPI. Here we present a study that compares shared sets of immune and tumor markers between the CODEX ${ }^{\infty}$ and Phenoptics ${ }^{\mathrm{TM}}$ platforms. This cross-platform comparison provides a conceptual framework for researchers to translate biomarker signatures from discovery to high-throughput translational studies.

Materials and Methods Serial sections of human formalin-fixed paraffin embedded non-small cell lung cancer (NSCLC) and tonsils were analyzed. An initial screen with a 28-plex CODEX $^{\circledast}$ antibody panel revealed multiple biomarkers of

Abstract P01.04 Figure 1

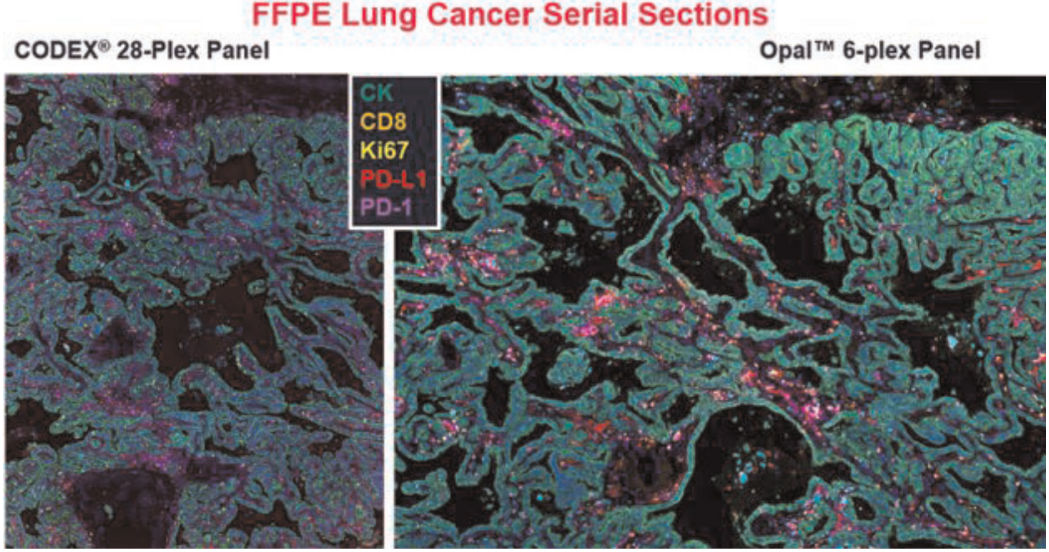

